\title{
Wide-field aurora imager onboard Fengyun satellite: Data products and validation
}

\author{
GuangXing Ding ${ }^{1}$, JiaWei $\mathrm{Li}^{2 *}$, XiaoXin Zhang ${ }^{2}$, Fei He ${ }^{3,4,5}$, LingPing He ${ }^{1,6}$, KeFei Song ${ }^{1,6}$, Liang Sun ${ }^{1}$, Shuang Dai ${ }^{1}$, \\ ShiJie Liu' ${ }^{1,6}$, Bo Chen ${ }^{1,6}$, Chao Yu², XiuQing Hu${ }^{2}$, SongYan Gu², ZhongDong Yang ${ }^{2}$, and Peng Zhang ${ }^{2}$ \\ ${ }^{1}$ Changchun Institute of Optics, Fine Mechanics and Physics, Chinese Academy of Sciences, Changchun 130033, China; \\ ${ }^{2}$ Key Laboratory of Space Weather, National Center for Space Weather, China Meteorological Administration, Beijing 100081, China; \\ ${ }^{3}$ Key Laboratory of Earth and Planetary Physics, Institute of Geology and Geophysics, Chinese Academy of Sciences, Beijing 100029, China; \\ ${ }^{4}$ Institutions of Earth Science, Chinese Academy of Sciences, Beijing 100029, China; \\ ${ }^{5}$ College of Earth and Planetary Sciences, University of Chinese Academy of Sciences, Beijing 100049, China; \\ ${ }^{6}$ State Key Laboratory of Applied Optics, Chinese Academy of Sciences, Changchun 130033, China
}

\section{Key Points:}

- The WAI can image the aurora with wide FOV, high sensitivity, and hightemporal and spatial resolutions in FUV wavelengths.

- The aurora data from WAI agree well with the SSUSI data in both auroraoval distribution and boundaries.

- The WAI observations can describe the aurora oval evolution with upstream solar wind parameters in detail.

Citation: Ding, G. X., Li, J. W., Zhang, X. X., He, F., He, L. P., Song, K. F., Sun, L., Dai, S., Liu, S. J., Chen, B., Yu, C., Hu, X. Q., Gu, S. Y., Yang, Z. D. and Zhang, P. (2021). Wide-field aurora imager onboard Fengyun satellite: Data products and validation. Earth Planet. Phys., 5(1), 73-78. http://doi.org/10.26464/epp2021003

\begin{abstract}
New observations of auroras based on the wide-field aurora imager (WAI) onboard Fengyun-3D (FY-3D) satellite are exhibited in this paper. Validity of the WAI data is analyzed by comparing auroral boundaries derived from WAI observations with results obtained from data collected by the Special Sensor Ultraviolet Spectrographic Imager (SSUSI) aboard the Defense Meteorological Satellite Program (DMSP F18). Dynamic variations of the aurora with the solar wind, interplanetary magnetic field (IMF) parameters, and the SYM-H index are also investigated. The comparison of auroral boundaries indicates that the WAI data are morphologically valid and suitable to the study of auroral dynamics. Effective responses to solar wind parameters indicate that the WAl data can be useful to monitor and predict the Earth's space weather. Since the configuration of aurora is a good indicator of the solar wind-magnetosphere-ionosphere (SW-M-I) coupling system, and can reflect the disturbance of the space environment, the WAI will provide important data to help us to study the physical processes in space.
\end{abstract}

Keywords: WAI; FY-3D; auroral dynamics; SSUSI; SW-M-I

\section{Introduction}

Auroras are produced by collisions between energetic magnetospheric particles precipitating into the polar region through the Earth's magnetic field lines and the atoms and molecules in the upper atmosphere (Meier, 1991). Variations of aurora are closely related to the dynamics of the ionosphere, the magnetosphere, and the solar wind, and the couplings between them. The configuration of the aurora is a good indicator of the solar wind-magnetosphere-ionosphere (SW-M-I) coupling system. Also, the aurora is highly dynamic during storms and substorms (Germany et al., 1998). Studying aurora is a valid method to explore the dynamics processes in the magnetosphere and the ionosphere.

Correspondence to: J. W. Li, lijw@cma.gov.cn

Received 02 MAR 2020; Accepted 28 AUG 2020.

Accepted article online 28 OCT 2020.

C) 2021 by Earth and Planetary Physics.
Studying the aurora phenomenon can help us to profoundly understand the processes in the solar-terrestrial space. For example, Liou and Sotirelis used auroral data from the Ultraviolet Imager (UVI) on the POLAR satellite to study responses of the northern winter polar cap to 28 auroral substorms; they found that the auroral breakup during substorms is preceded by inflation of the polar cap and followed by deflation of the polar cap (Liou and Sotirelis, 2016). Milan utilized the auroral images obtained by the Wideband Imaging Camera (WIC) onboard the IMAGE satellite to track changes in the polar cap area and to quantify the amount of open flux in the magnetosphere (Milan et al., 2012). Zhang and Paxton used auroral data from the Global Ultraviolet Imager (GUVI) onboard the TIMED satellite to construct an empirical $K p$-dependent global auroral model (Zhang and Paxton, 2008). Luan utilized SSUSI/DMSP data to study the dependences of the auroral energy flux distribution over the nightside oval with seasonal variety, geomagnetic parameters, and solar activity (Luan XL et al., 2018). Ding used GUVI/TIMED and SSUSI/DMSP auroral data to develop 
an automatic auroral boundary determination algorithm (Ding GX et al., 2017).

The observation of auroras has gone through a long period. Auroral configuration has been described by unaided human visual observation, and by instrumental observation from ground-based stations, and more recently via imaging from space. Imaging the auroral oval from space provides spatial/temporal information of the precipitation particles that allows these particles to be mapped back to different source regions in the magnetosphere, which allows us to understand the energy transportation processes (Germany et al., 1994a, b). Since the $20^{\text {th }}$ century, more and more satellites have been sent to space to obtain more precise spatial data; the aurora is especially important among the observed phenomena. For example, the UVI/POLAR, the WIC/IMAGE, the GUVI/TIMED, and the SSUSI/DMSP (Paxton et al., 2004; Sotirelis et al., 2013). The WAI, developed by the Institute of Optics, Fine Mechanics, and Physics, Chinese Academy of Sciences, is aboard the FY-3D satellite, which was launched on 15 November 2017; on 8 December 2017, it began routinely acquiring scientific data. Since then, the ground application system of FY-3D has accumulated a large number of auroral images.

Both the GUVI and SSUSI spectrographic instruments are of the scanning type, with limited Field of View (FOV); they cannot capture the global auroral ovals due to the low altitude of their sunsynchronous satellite orbits (Paxton et al., 1992, 1993, 2004). The WAI has a novel design that allows it to get relatively wide-area auroral images at low altitudes, compared to whose from the SSUSI. The purpose of this paper is to verify the validity of WAI data by comparing auroral configurations derived from WAI data with the observations of SSUSI, and by investigating the auroral evolution with variable solar wind. The system design, instrument development, and the data processing algorithm that transfer photon signals to disk images projected in geographic/geomagnetic grids on the reference sphere of WAI have been introduced in Zhang's paper (Zhang XX et al., 2019).

\section{Materials and Methods}

\subsection{Data Sets}

The auroral images used in this study were obtained by the WAI/FY-3D and the SSUSI/DMSP F18. The FY-3D satellite operates in a sun-synchronous orbit with an orbital height of about $836 \mathrm{~km}$. The instantaneous FOV of the WAI optical system is $130^{\circ} \times 10^{\circ}$ $\left(130^{\circ}\right.$ corresponds to the cross-track direction and $10^{\circ}$ corresponds to the along-track direction) with operational wavelength range $140-180 \mathrm{~nm}$. In scanning mode, the WAI can achieve a total FOV of $130^{\circ} \times 130^{\circ}$, which allows relatively complete auroral images to be obtained. Since the launch of the FY-3D on 15 November 2017, the WAI has accumulated and returned a large number of auroral images. The WAI data used in this paper are provided by the ground application system of the FY-3D. The data processing algorithm operated in the ground application system is described in Zhang's paper (Zhang XX et al., 2019).

Another category of auroral images, used here as a comparison for the images obtained from WAI data, were obtained by the SSUSI instruments (Paxton et al., 2004; Sotirelis et al., 2013). The
DMSP satellites were launched into circular polar orbits with orbital height of $840 \mathrm{~km}$. From F16 through F18, the DMSP satellites were equipped with SSUSI; SSUSI data are available from 2004 on. The SSUSI optical system includes an imaging spectrograph with instantaneous FOV of $11.8^{\circ}$, with five channels whose wavelengths are between 115 and $180 \mathrm{~nm}$ (including the LBH band from 140 to $180 \mathrm{~nm}$ ), and equipped with cross track scanning mode. The spectrographic imager was designed to provide quantitative observations and interpretations of the Earth's airglow and auroral emissions in FUV. The SSUSI auroral images used in this paper were obtained from the websites of http://ssusi. jhuapl.edu/.

The solar wind and interplanetary magnetic field (IMF) parameters used in this paper were provided by NASA OMNI Web. The three components of IMF, the solar wind density and velocity, and the solar wind dynamic pressure, are in $1 \mathrm{~min}$ resolution. Since the data were obtained at the nose of the magnetopause, it is necessary to consider the delay that is known to occur as a phenomenon propagates to the ionosphere. The propagation times are estimated to be the sum of 5 min for traversing the magnetosheath and 2 min Alfvén transit time to reach the ionosphere (Liou et al., 1998).

\subsection{Comparison with DMSP}

The SSUSU auroral data have been proverbial in studies of space dynamics transversion. In this section, we perform detailed comparisons between SSUSI observations and WAI observations in order to establish the validity and accuracy of the WAI results. These comparisons include auroral shapes and boundaries.

To examine the consistency of the auroral radiation distribution between WAI and SSUSI, we compared the adjoining time observations of the two instruments. The geomagnetic activity was basically quiet on 30 August 2018, with a maximum $K p$ of 2 . The auroral observations at about 00:15 (UT) are shown in Figure 1. The red asterisks in (a) and black asterisks in (b) show the equatorward boundaries that were extracted with the fuzzy local information C-means clustering segmentation algorithm (FLICM) (Yang QJ et al., 2013; Ding GX et al., 2017). The FLICM algorithm incorporates local spatial and gray level information in a fuzzy way in order to preserve robustness and noise insensitiveness, by adding a novel fuzzy factor

$$
G_{k i}=\sum_{\substack{j \in N_{i} \\ i \neq j}} \frac{1}{d_{i j}+1}\left(1-u_{k j}\right)^{m}\left\|x_{j}-v_{k}\right\|^{2}
$$

to the squared error objective function

$$
J_{m}=\sum_{i=1}^{N} \sum_{k=1}^{c}\left[u_{k i}^{m}\left\|x_{i}-v_{k}\right\|^{2}+G_{k i}\right]
$$

where $N$ is the total number of pixels; $c$ is the number of clusters with $2 \leq c<N$ (in this paper, we set $c$ to be 6 ); $x_{i}$ is the gray level of the $i^{\text {th }}$ pixel that is the center of the local window (e.g., $3 \times 3$ pixels); $v_{k}$ is the prototype of the center of cluster $k ; u_{k i}$ is the degree of the membership of $x_{i}$ in the $k^{\text {th }}$ cluster; $m$ is a weighting exponent on each fuzzy membership that determines the amount of fuzziness of the resulting classification, and is usually set to be 2 
(a) WAI FY-3D 2018/08/30 00:15

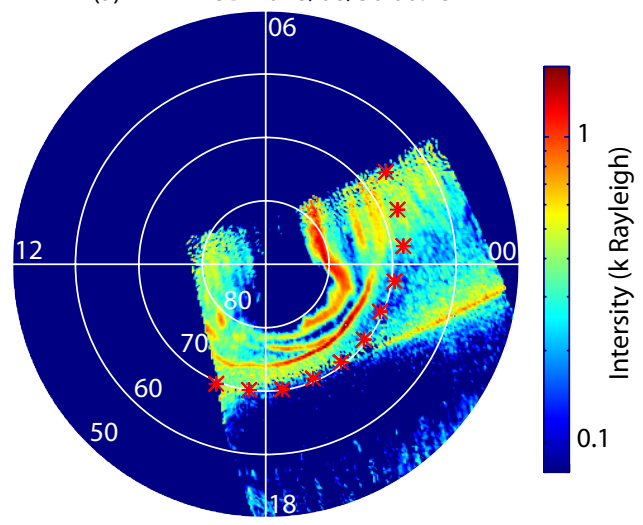

(b) DMSP F18 2018/08/30 00:00

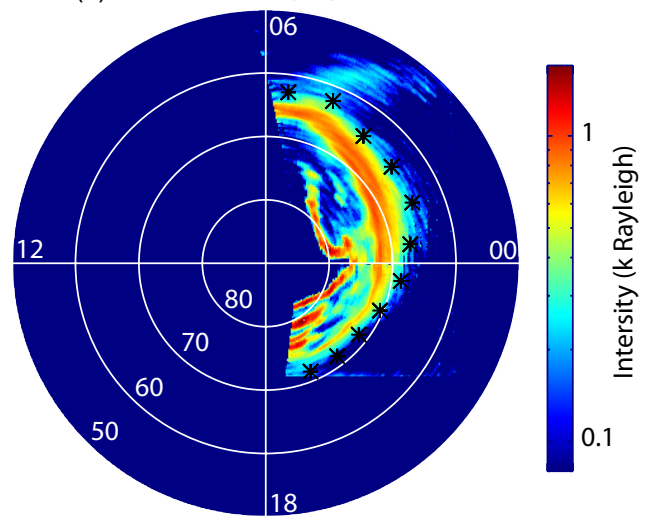

Figure 1. Aurora images observed by (a) FY-3D WAI and (b) DMSP F18 SSUSI in the southern hemisphere at 00:15 and 00:00 on 30 August 2018. Two images have a time interval of 15 minutes. The red asterisks in (a) and black asterisks in (b) are the equatorward boundaries extracted by the FLICM algorithm. Midnight is on the right and noon is on the left.

(Bezdek, 1981); $N_{i}$ stands for the set of neighbors falling into a local window around pixel $x_{i}$ and $d_{i j}$ is the spatial Euclidean distance between pixels $i$ and $j$. The accuracy of the auroral boundaries extracted by FLICM has been verified in several studies (Yang QJ et al., 2013; Ding GX et al., 2017). It can be seen from Figure 1 that the auroral boundaries of WAI origin are quite similar to those from SSUSI data.

The comparison of auroral images from WAI and SSUSI (given in Figure 2) testifies to the consistency of their detection of auroral morphology. In order to eliminate differences between the outputs of two instruments, normalization of data between the two instruments was done before merging. For the locations that were observed by both WAI and SSUSI, the average intensities were calculated for each, IWAI for WAI and ISSUSI for SSUSI, respectively. Then the normalization factor $I_{\text {SSUSI }} / I_{\text {WAI }}$ was used to normalize the

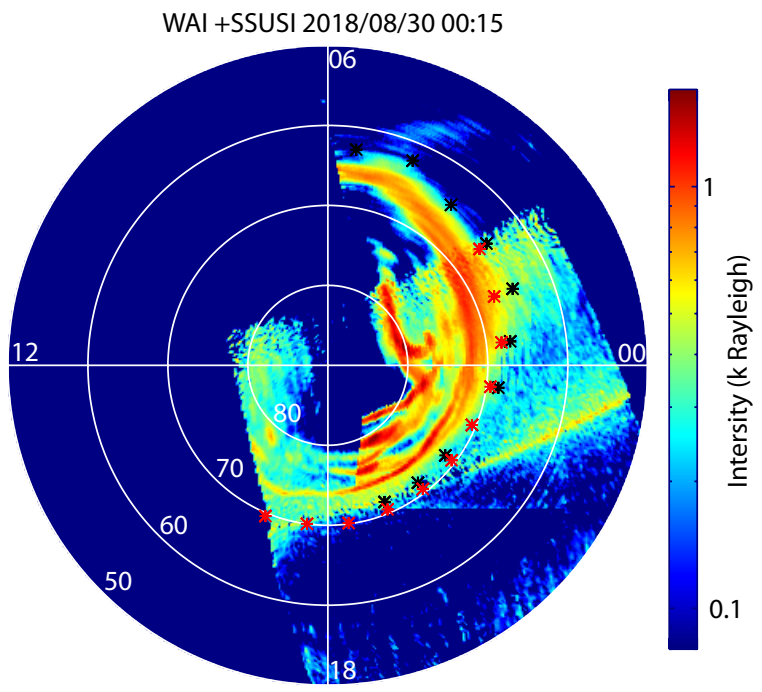

Figure 2. The superposition of aurora images derived from WAI and from SSUSI observations. The superposed auroral images come from Figure 1. The normalization factor $I_{\text {SSUSI }} / I_{\text {WAI }}$ has been used to normalize the WAI data to the level of SSUSI data. The red and black asterisks are the equatorward boundaries.
WAI data to the level of SSUSI; that is, all the pixels in the WAI image are multiplied by $I_{\text {SSUSI }} / I_{\text {WAI }}$. The red asterisks in Figure 2 are the auroral equatorward boundaries derived from WAI observations; the black asterisks are the auroral equatorward boundaries derived from SSUSI data. Figure 2 demonstrates that the auroral morphologies of WAI and SSUSI are in good agreement. The auroral radiation distribution tendencies and the local features of the two observations also show quite fine agreement. The locations of the equatorward boundaries are basically identical. These indicate that the WAI observations are highly consistent with those of the SSUSI.

A median geomagnetic storm, with the maximum $K p$ of 6 , erupted between 5-7 May, 2018. The observations of auroral ovals at about 20:44 UT, 5 May 2018 are shown in Figure 3, in which the red and black asterisks are the equatorial boundaries extracted with the FLICM algorithm. Figure 4 superimposes the auroral images of Figure 3. It can also be seen from the figures that the distribution tendencies of the auroral radiation and the local features of the two observations show quite fine agreement. From the two cases, the trends of auroral boundaries and the similarity of the featured regions can demonstrate that the observations of WAI have fine consistency with those of SSUSI.

Furthermore, in order to verify the match between auroral images from WAI and observations from SSUSI, southern hemisphere auroral images from June 15, 2018 to June 24, 2018 that were observed by both WAI and SSUSI were selected for statistical analysis. Auroral images from WAI during this time period were matched one at a time with the corresponding images from SSUSI. A time window of 10 min was chosen to select the auroral images from the two instruments to minimize temporal evolution of the auroral oval between the two observations. We have assumed that the auroral oval is sufficiently stable for meaningful comparison at the 10 min time scale; however, this may not be true of observations made during the main phase of storms or the expansion phase of substorms. Once an image pair satisfying this criterion was determined, the two data sets were mapped into the same grid of magnetic latitude (MLAT) and magnetic local time $(\mathrm{MLT})$ in the corrected (altitude-adjusted) geomagnetic coordin- 
(a) WAI FY-3D 2018/05/05 20:44

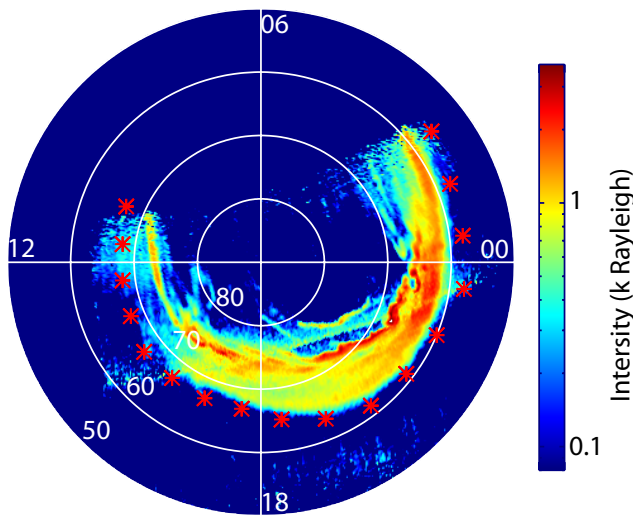

(b) DMSP F18 2018/05/05 20:40

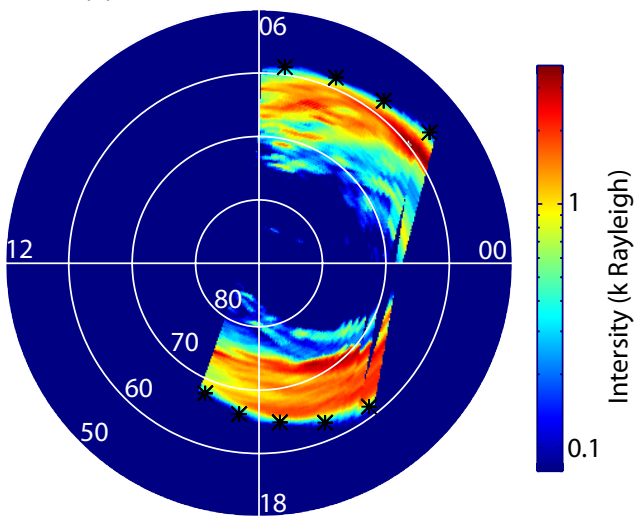

Figure 3. Aurora images observed by FY-3D WAI and DMSP F18 SSUSI in the southern hemisphere at 20:44 and 20:40 on 5 May 2018. The annotations are same as for Figure 1.

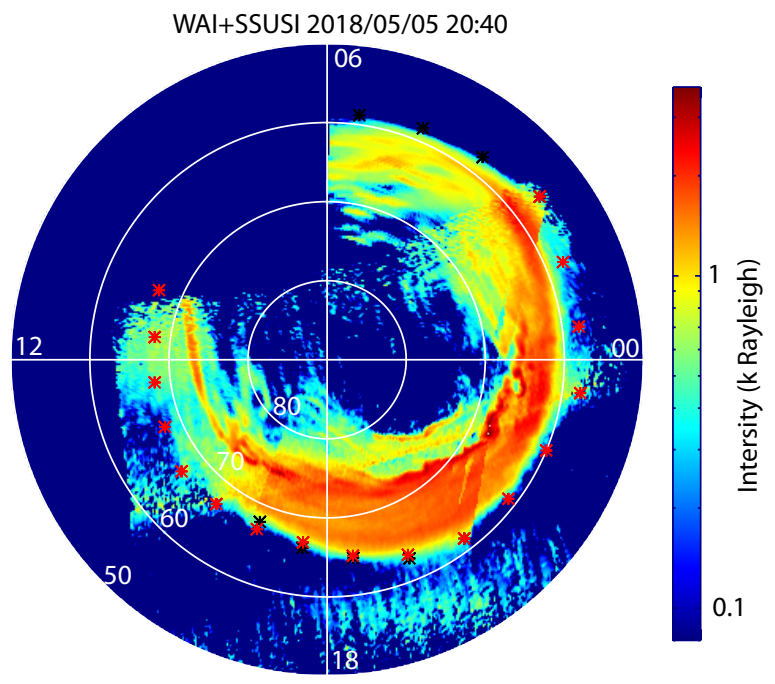

Figure 4. Superimposition of WAI and SSUSI aurora images from Figure 3. The annotations are the same as for Figure 2.

ates. Then the auroral equatorward boundaries were extracted from the two data sets, using the FLICM algorithm. The statistics of two kinds of boundary points are shown in Figure 5. As can be seen, there are 347 pairs of boundary points in total and the correlation coefficient is 0.927 . All the scatters are linearly fitted to

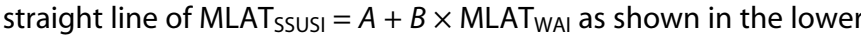
part in Figure 5. $A$ is $1.310, B$ is 0.983 and the uncertainties of $A$ and $B$ are 1.300 and 0.020 . The gray solid line indicates MLAT $_{\text {SSUSI }}=$ MLAT $_{\text {WAl; }}$ that is, MLAT SSUSI $_{\text {and MLAT }}$ WAI agree perfectly; the dashed lines are plotted at MLAT $T_{\text {SSUSI }}=\operatorname{MLAT}_{W A I} \pm 3^{\circ}$. The standard deviations between MLAT $_{\text {SSUSI }}$ and MLAT WAI is $1.038^{\circ}$, and $68.876 \%, 98.559 \%, 98.847 \%$ of the scatters within $\pm 1^{\circ}$, $\pm 2^{\circ}, \pm 3^{\circ}$, respectively. In summary, the boundaries derived from WAI observations agree very well with those from SSUSI data.

\subsection{Variations with Solar Wind}

The final driving sources of auroral variations are the solar wind and the embedded IMF. Especially when the IMF is southward, the magnetic field of the solar wind begins to reconnect to the Earth's magnetic field. Energetic particles carried by the solar wind will

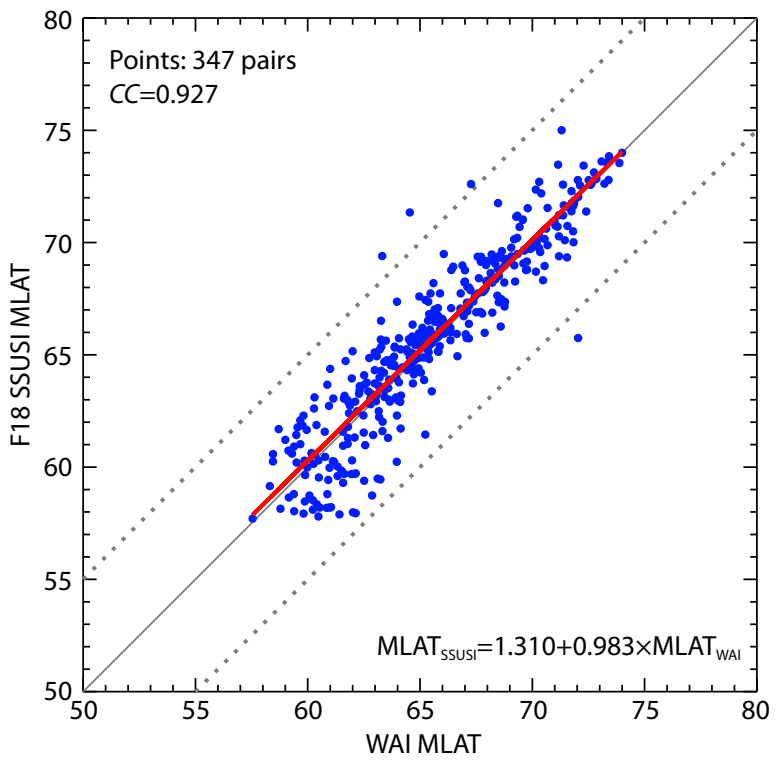

Figure 5. Comparison between auroral equatorward boundaries of WAI and auroral equatorward boundaries of SSUSI. The grey solid line indicates $M_{\text {LAT }}$ SSUSI $_{\text {I }}$ MLAT $_{\text {WAI }}$, and the dashed lines are plotted at $M_{\text {SASUSI }}=$ MLAT $_{W A I} \pm 3^{\circ}$. The red solid lines are the linear fitting of $M L A T_{\text {WAI }}$ to MLAT $T_{\text {SSUSI }}$ with the correlation coefficient shown in the top left corner.

enter the polar upper atmosphere along the Earth's magnetic field lines and then collide with atmospheric particles to trigger the breakup of the aurora.

The variations of solar wind and IMF parameters on 25 August 2018 are shown in Figure 6. It is shown that there was a change of IMF $B_{z}$ from north to south at about 15:30 (UT). Figure 7 presents the auroral images from WAI before and after the southward turning of the IMF. The solar wind and geomagnetic parameters corresponding to each auroral image are annotated in Figure 6 in different colors.

At 10:18, the $B_{z}$ was $7.63 \mathrm{nT}$ with a north direction, the solar wind velocity was $404 \mathrm{~km} / \mathrm{s}$, and the SYM-H was $15 \mathrm{nT}$. The auroral image observed by the WAl corresponding to this time is shown in 


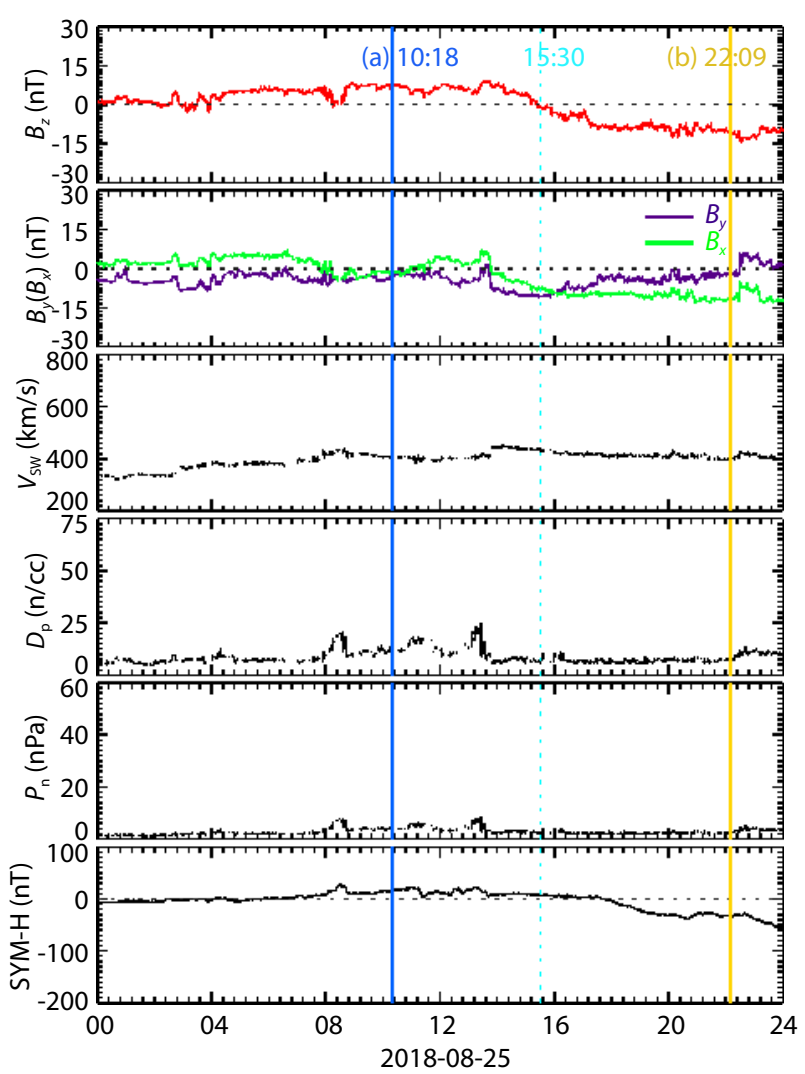

Figure 6. The fluctuation of IMF parameters, solar wind parameters, and SYM-H on 25 August 2018. The IMF $B_{z}$ turns to be south at 15:30, marked with the dotted line. Before the turning point (a) and after the turning point (b) are chosen to study the evolution of aurora. The auroral images observed by WAI are displayed in Figure 7.

(a) $10: 182018 / 08 / 25$

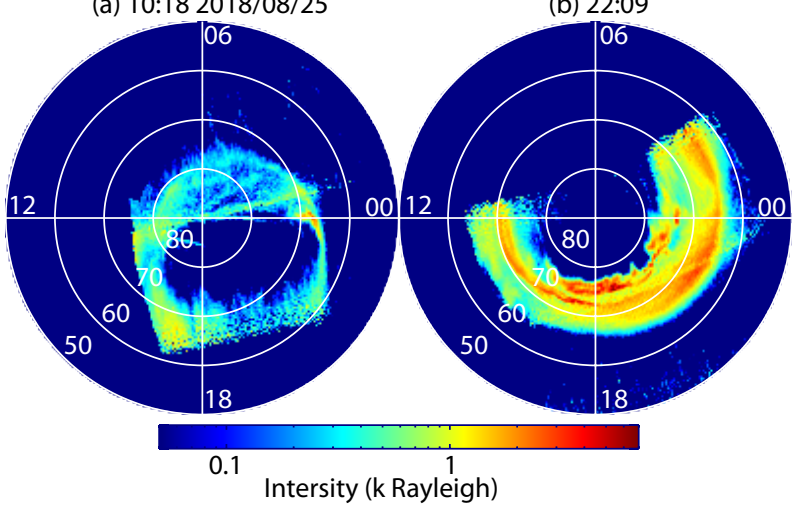

Figure 7. The auroral images observed by the WAI before and after southward turning of IMF. (a) Auroral image obtained at 10:18 UT. (b) Auroral image obtained at 22:09 UT.

Figure 7a. Under the prevailing conditions of quiet solar wind and geomagnetic conditions, the auroral activity was weak, characterized by low emission intensity and an auroral oval of small size.

When the IMF turned to become southward after 15:30 UT, the geomagnetic activity became disturbed, and the SYM-H index began gradually to decrease. At 22:09 UT, the auroral image obtained by the WAI indicates that both the poleward and equator- ward boundaries of the auroral oval had significantly expanded and the auroral intensity had risen significantly.

\section{Results}

The WAI onboard the FY-3D can image the aurora with wide FOV, high sensitivity, and high temporal and spatial resolution in FUV wavelengths. The intensity and distribution of the aurora directly reflect geomagnetic activities, the dynamics of the energetic particles near the Polar Regions, and the interaction between the solar wind and the ionosphere/magnetosphere. Based on comparison with the observations of SSUSI/DMSP, it can be concluded that WAI data agree well with SSUSI data in describing both the aurora oval distribution and auroral boundaries. These comparison results indicate that the observations between WAI and SSUSI have fine consistency, and that the WAI data have good quality.

WAI data have thus been used in this paper to investigate the evolution of auroral morphology during geomagnetic events. We have verified that WAI observations can describe in detail the aurora oval evolution with upstream solar wind parameters. We conclude that WAI data can be used effectively to monitor and predict space weather.

\section{Conclusions}

In this paper, the auroral images of the WAI onboard FY-3D are compared with those of the SSUSI/DMSP. The evolution of auroras with upstream solar wind parameters is also investigated. We report that the WAI data have fine agreement with the SSUSI data, and can depict the aurora morphology during periods of geomagnetic activity. WAI data will play an important role in monitoring space weather and in warning operations at the National Center of Space Weather (National Satellite Meteorological Center) of China, and can also be helpful in study of the coupling of the SWM-I system.

\section{Acknowledgments}

All the authors declare that they have no conflict of interest. This work was supported in part by the National Science Foundation of China (41327802, 41774152, 41674155 and 41931073), in part by Youth Innovation Promotion Association of the Chinese Academy of Sciences (2017258), and by the Strategic Priority Program on Space Science, Chinese Academy of Sciences, Grant No. XDA15350203. The authors would like to thank NASA and WDC for geomagnetism data and Kyoto World Data Center for providing the geomagnetic index. The WAI data are provided by the ground application system of FY-3D.

\section{References}

Bezdek, J. C. (1981). Pattern Recognition with Fuzzy Objective Function Algorithms. Boston: Springer. https://doi.org/10.1007/978-1-4757-0450-1

Ding, G. X., He, F., Zhang, X. X., and Chen, B. (2017). A new auroral boundary determination algorithm based on observations from TIMED/GUVI and DMSP/SSUSI. J. Geophys. Res., 122(2), 2162-2173. https://doi.org/10.1002/2016JA023295

Germany, G. A., Torr, D. G., Richards, P. G., Torr, M. R., and John, S. (1994a). Determination of ionospheric conductivities from FUV auroral emissions. J. 
Geophys. Res., 99(A12), 23297-23305. https://doi.org/10.1029/94JA02038

Germany, G. A., Torr, M. R., Torr, D. G., and Richards, P. G. (1994b). Use of FUV auroral emissions as diagnostic indicators. J. Geophys. Res., 99(A1), 383-388. https://doi.org/10.1029/93JA02357

Germany, G. A., Parks, G. K., Ranganath, H., Elsen, R., Richards, P. G., Swift, E., Spann, J. F., and Brittnacher, M. (1998). Analysis of auroral morphology: substorm precursor and onset on January 10, 1997. Geophys. Res. Lett., 25(15), 3043-3046. https://doi.org/10.1029/98GL01220

Liou, K., Newell, P. T., Meng, C. I., Brittnacher, M., and Parks, G. (1998). Characteristics of the solar wind controlled auroral emissions. J. Geophys. Res., 103(A8), 17543-17557. https://doi.org/10.1029/98JA01388

Liou, K., and Sotirelis, T. (2016). Response of northern winter polar cap to auroral substorms. Geophys. Res. Lett., 43(9), 4098-4105. https://doi.org/10.1002/2016GL068039

Luan, X. L., Zhou, S., and Dou, X. K. (2018). Auroral energy flux distribution over the nightside auroral oval observed by the DMSP F16/SSUSI: seasonal, geomagnetic, and solar activity dependences. J. Geophys. Res., 123(5), 4457-4466. https://doi.org/10.1029/2017JA023970

Meier, R. R. (1991). Ultraviolet spectroscopy and remote sensing of the upper atmosphere. Space Sci. Rev., 58(1), 1-185. https://doi.org/10.1007/BF01206000

Milan, S. E., Gosling, J. S., and Hubert, B. (2012). Relationship between interplanetary parameters and the magnetopause reconnection rate quantified from observations of the expanding polar cap. J. Geophys. Res., 117(A3), A03226. https://doi.org/10.1029/2011JA017082

Paxton, L. J., Meng, C. I., Fountain, G. H., Ogorzalek, B. S., Darlington, E. H., Gary, S. A., Goldsten, J. O., Kusnierkiewicz, D. Y., Lee, S. C., ... Smith, B. E. (1992). Special sensor ultraviolet spectrographic imager: an instrument description. In Proceedings of SPIE Instrumentation for Planetary and Terrestrial
Atmospheric Remote Sensing (pp. 2-16). San Diego, CA, United States: SPIE. https://doi.org/10.1117/12.60595

Paxton, L. J., Meng, C. I., Fountain, G. H., Ogorzalek, B. S., Darlington, E. H., Gary, S. A., Goldsten, J. O., Kusnierkiewicz, D. Y., Lee, S. C., ... Daniell, R. E. Jr. (1993). SSUSI-Horizon-to-horizon and limb-viewing spectrographic imager for remote sensing of environmental parameters. In Proceedings of SPIE Ultraviolet Technology IV. San Diego, CA, United States: SPIE. https://doi.org/10.1117/12.140846

Paxton, L. J., Christensen, A. B., Morrison, D., Wolven, B., Kil, H., Zhang, Y. L., Ogorzalek, B. S., Humm, D. C., Goldsten, J. O., ... Meng, C. I. (2004). GUVI: a hyperspectral imager for geospace. In Proceedings of SPIE Instruments, Science, and Methods for Geospace and Planetary Remote Sensing (pp. 228449). Honolulu, Hawai'i, United States: SPIE. https://doi.org/10.1117/12.579171

Sotirelis, T., Korth, H., Hsieh, S. Y., Zhang, Y. L., Morrison, D., and Paxton, L. (2013). Empirical relationship between electron precipitation and farultraviolet auroral emissions from DMSP observations. J. Geophys. Res., 118(3), 1203-1209. https://doi.org/10.1002/jgra.50157

Yang, Q. J., Liang, J. M., Liu, J. M., Hu, J. Z., and Hu, H. Q. (2013). A method for automatic identification of substorm expansion phase onset from UVI images. Chin. J. Geophys. (in Chinese), 56(5), 1435-1447. https://doi.org/10.6038/cjg20130502

Zhang, X. X., Chen, B., He, F., Song, K. F., He, L. P., Liu, S. J., Guo, Q. F., Li, J. W., Wang, X. D., ... Wang, J. S. (2019). Wide-field auroral imager onboard the Fengyun satellite. Light: Sci. Appl., 8, 47. https://doi.org/10.1038/s41377-0190157-7

Zhang, Y., and Paxton, L. J. (2008). An empirical Kp-dependent global auroral model based on TIMED/GUVI FUV data. J. Atmos. Terr. Phys., 70(8-9), 1231-1242. https://doi.org/10.1016/j.jastp.2008.03.008 